\title{
SPONGENT: The Design Space of Lightweight Cryptographic Hashing
}

\author{
Andrey Bogdanov, Miroslav Knežević, Gregor Leander, Deniz Toz, Kerem Varıcı, and Ingrid Verbauwhede
}

\begin{abstract}
The design of secure yet efficiently implementable cryptographic algorithms is a fundamental problem of cryptography. Lately, lightweight cryptography - optimizing the algorithms to fit the most constrained environments - has received a great deal of attention, the recent research being mainly focused on building block ciphers. As opposed to that, the design of lightweight hash functions is still far from being well-investigated with only few proposals in the public domain. In this article, we aim to address this gap by exploring the design space of lightweight hash functions based on the sponge construction instantiated with PRESENT-type permutations. The resulting family of hash functions is called SPONGENT. We propose 13 SPONGENT variants - for different levels of collision and (second) preimage resistance as well as for various implementation constraints. For each of them we provide several ASIC hardware implementations - ranging from the lowest area to the highest throughput. We make efforts to address the fairness of comparison with other designs in the field by providing an exhaustive hardware evaluation on various technologies, including an open core library. We also prove essential differential properties of SPONGENT permutations, give a security analysis in terms of collision and preimage resistance, as well as study in detail dedicated linear distinguishers.
\end{abstract}

Index Terms-hash function, lightweight cryptography, low-cost cryptography, low-power design, sponge construction, PRESENT, SPONGENT, RFID.

\section{INTRODUCTION}

\subsection{Motivation and related work}

As crucial applications go pervasive, the need for security in RFID and sensor networks is dramatically increasing, which requires secure yet efficiently implementable cryptographic primitives. The most important types of cryptographic algorithms include secret-key ciphers (mainly used to provide data secrecy) and hash functions (multifaceted primitives with the primary usage of data authenticity). In such constrained environments, the area and power consumption of a primitive usually comes to the fore and standard algorithms are often prohibitively expensive to implement.

Once this research problem was identified, the cryptographic community designed a number of tailored lightweight cryptographic algorithms to specifically address this challenge. Stream ciphers (that is, ciphers that process the data in a bitwise manner) like Trivium [10], and block ciphers (i.e. ciphers that operate on blocks of data simultaneously) like KATAN/KTANTAN [11], and PRESENT [5] emerged - to mention only a small selection of the lightweight designs.

It was not until recently though that some significant work on lightweight hash functions has been also per-

- Andrey Bogdanov, Miroslav Knežević, Deniz Toz, Kerem Varıcı, and Ingrid Verbauwhede are with KU Leuven, ESAT/COSIC and IBBT, Belgium. E-mail: \{andrey.bogdanov, deniz.toz, kerem.varici, ingrid.verbauwhede\}@esat.kuleuven.be.

- Miroslav Knežević is with NXP Semiconductors, Leuven, Belgium. E-mail: miroslav.knezevic@nxp.com.

- Gregor Leander is with DTU Mathematics, Technical University of Denmark.E-mail: g.leander@mat.dtu.dk. formed: [6] describes ways of using the PRESENT block cipher in hashing modes of operation and [1] and [14] take the approach of designing a dedicated lightweight hash function based on a sponge construction [9], [2] resulting in two hash functions QUARK and PHOTON.

A hash function is a function that maps a bit sequence of arbitrary length to a fixed-length output. As a rule, three security requirements are mentioned for a hash function. First, it should be preimage resistant: Given an output (hash value), it should be hard to find an input (message) that would map to this output. Second, it should be second-preimage resistance: Given a hash value and a corresponding message, it should be difficult to find another message with the same hash value. Finally, it should be collision resistant: It should be infeasible to find two messages with the same hash value.

Among the most prominent security applications targeted by a lightweight hash function are (including the ones requiring preimage security only and collision security only):

- Lightweight signature schemes: ECC (Elliptic Curve Cryptography that can be used for both producing digital signatures and public-key encryption) over $\mathbb{F}_{2^{163}}$ is implementable with just 11.904 GE without key storage after synthesis and around 15.000 GE on a chip [16]. For comparison, the smallest published implementation [18] of the NISTstandardized state-of-the-art hash function SHA256 requires 8.588 GE. The reportedly most compact SHA-3 finalists BLAKE and Grøstl need 13.560 GE [17] and 14.620 GE [24], respectively, to our best knowledge (SHA-3 is the competition launched by 
NIST to eventually update the U.S. hash standard). Hence, adding a hashing engine based on SHA-2 to a lightweight ECC implementation would increase its area by at least $50 \%$ and adding BLAKE or Grøstl would double the area occupation.

- RFID security protocols often rely on hash functions. Some of the applications require collision resistance and some of them do not, just needing preimage security. An interesting case is constituted by keyed message authentication codes (MAC) often used in this context. Here, a lightweight hash function can require less area than a lightweight block cipher in a MAC mode at a fixed level of offline and online security. MACs can be also designed using sponge primitives [3].

- Random number generation in hardware is used for ephemeral key generation (a temporary key that is used only once and discarded after usage) in public-key schemes, producing random input for cryptographic protocols, and for masking schemes in implementations with protection against sidechannel attacks. This frequently needs a preimageresistant hash function. Using a hash function for pseudorandom number generator (PRNG), given a seed, provides backward security which a block cipher based PRNG (e.g. in OFB mode) does not: Once the key is leaked e.g. through a side-channel attack, the adversary can compute the previous outputs of the block cipher based PRNG. Moreover, the postprocessing of a physical random number generator sometimes includes a preimage-resistant hash function.

- Post-quantum signature schemes can be built upon a hash function using Merkle trees. There have been several attempts to efficiently implement it [23]. Having a lightweight hash function allows to derive a more compact implementation of the Merkle signature scheme.

However, while for multiple block ciphers, designs have already closely approached the minimum ASIC hardware footprint theoretically attainable, it does not seem the case for some recent lightweight hash functions so far.

\subsection{Design considerations for lightweight hashing}

The footprint of a hash function is mainly determined by

1) the number of state bits (incl. the key schedule for block cipher based designs) as well as

2) the size of functional and control logic used in a round function.

For highly serialized implementations (usually used to attain low area and power), the logic size is normally rather small and the state size dominates the total area requirements of the design. Among the recent hash functions, QUARK, while using novel ideas of reducing the state size to minimize (1), does not appear to provide the smallest possible logic size, which is mainly due to the Boolean functions with many inputs used in its round transform. In contrast to that, SPONGENT keeps the round function very simple which reduces the logic size close to the smallest theoretically possible, thus, minimizing (2) and resulting in a significantly more compact design.

As shown in [6], using a lightweight block cipher in a hashing mode (single block length such as Davies-Meyer or double block length such as Hirose) is not necessarily an optimal choice for reducing the footprint, the major restriction being the doubling of the datapath storage requirement due to the feed-forward operation.

At the same time, no feed-forward is necessary for the sponge construction, which is the design approach of choice in this work. In a permutation-based sponge construction, let $r$ be the rate (the number of bits input or output per one permutation call), $c$ be the capacity (internal state bits not used for input or output), and $n$ be the hash length in bits.

\subsection{Contributions}

This article proposes the family of sponge-based lightweight hash functions SPONGENT with a smaller footprint than most existing dedicated lightweight hash functions: PRESENT in hashing modes and QUARK. Its area is comparable to that of PHOTON. However, we would like to stress that a fair comparison of spongebased hash functions in terms of their area requirements is a challenging task. It is not only due to the fact that the area occupation is highly dependent on the implementation, technology and tools used but also the very parameterization of sponge designs has an immense impact on their hardware performance. By just slightly changing the rate of a sponge-based hash function, one can either make the resulting design smaller and slower or larger and faster. The design of PHOTON opts for a higher rate and a slightly larger area occupation, while SPONGENT chooses for a lower rate and somewhat lower area requirements. With a proper choice of parameters, one can actually turn the situation and make SPONGENT faster though bigger and РНОТОN slower but smaller. However, to keep the comparison feasible and representable, we prefer to stick to the original designs in this paper. To address the discrepancies in the hardware design flows, we provide implementation figures for SPONGENT on four different technologies. In order to make the comparisons fairer, we also provide the hardware figures for SPONGENT, PHOTON and QUARK based on an open core library.

For some SPONGENT variants, similarly to QUARK and PHOTON, a part of their advantage in terms of low area comes from a reduced level of second preimage security, while maintaining the standard level collision resistance. The other SPONGENT variants attain the standard preimage, second preimage and collision security, while having area requirements much lower than those of SHA-1, 
SHA-2, and SHA-3 finalists. This design subspace has not been specifically addressed by any previous concrete lightweight hash function proposal. Whereas we note that the design ideas of PRESENT in hashing modes, QUARK and PHOTON might be extended to any set of security parameters.

To explore the design space of lightweight hashing, we propose to instantiate the sponge construction with a PRESENT-type permutation. The resulting construction is called SPONGENT and we refer to its various parameterizations as SPONGENT- $n / c / r$ for different hash sizes $n$, capacities $c$, and rates $r$. SPONGENT is a hermetic sponge, i.e., we do not allow the underlying permutation to have any structural distinguishers. More precisely, for five different hash sizes of $n \in\{88,128,160,224,256\}$, covering most security applications in the field, we consider (up to) three types of preimage and secondpreimage security levels:

- Full preimage and second-preimage security. The standard security requirements for a hash function with an $n$-bit output size are collision resistance of $2^{n / 2}$ as well as preimage and second-preimage resistance of $2^{n}$. For this, in SPONGENT, we set $r=n$ and $c=2 n$ to obtain SPONGENT-88/176/88, SPONGENT-128/256/128, SPONGENT-160/320/160, SPONGENT-224/448/224, and SPONGENT-256/512/256.

- Reduced second-preimage security. The design of [1] as well as the papers [2], [3], [9] convincingly demonstrate that a permutation-based sponge construction can allow to almost halve the state size for $n \geq c$ and reasonably small $r$. In this case, the preimage and second-preimage resistances are reduced to $2^{n-r}$ and $2^{c / 2}$, correspondingly, while the collision resistance remains at the level of $2^{c / 2}$. In most embedded scenarios, where a lightweight hash function is likely to be used, the full second-preimage security is not a necessary requirement. For relatively small rate $r$, the loss of preimage security is limited. So we take this parametrization in the design of the smallest SPONGENT variants with $n \approx c$ for small $r$ and obtain SPONGENT-88/80/8, SPONGENT-128/128/8, SPONGENT-160/160/16, SPONGENT-224/224/16, and SPONGENT-256/256/16. These five SPONGENTvariants were published in a shortened conference version [4] of this article.

- Reduced preimage and second-preimage security. In some applications, the collision security is of concern only and one can abandon the requirement of preimage security to be close to $2^{n}$. In a permutation-based sponge, going for $c=n$ and $r=n / 2$, results in the reduction of both the preimage security and second-preimage security to $2^{n / 2}$, while maintaining the full collision security of $2^{n / 2}$. On the implementation side, this parameterization can yield a favorable ratio between the rate and the permutation size which reduces the time-

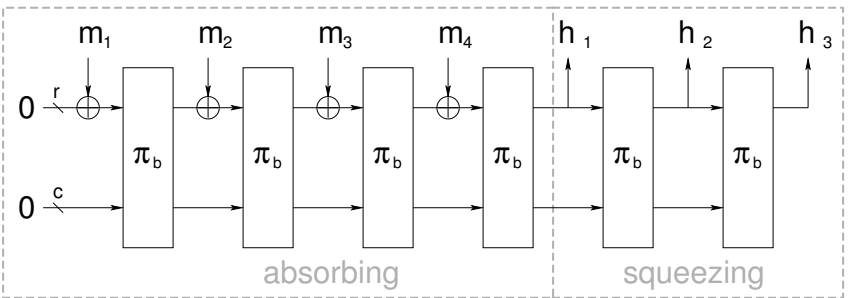

Fig. 1. Sponge construction based on a $b$-bit permutation $\pi_{b}$ with capacity $c$ bits and rate $r$ bits. $m_{i}$ are $r$-bit message blocks. $h_{i}$ are parts of the hash value.

area product. We use this approach in the design of SPONGENT-160/160/80, SPONGENT-224/224/112, and SPONGENT-256/256/128.

The group of all SPONGENT variants with the same output size of $n$ bits is referred to as SPONGENT- $n$. The SPONGENT- 88 functions are designed for extremely restricted scenarios and low preimage security requirements. They can be used e.g. in some RFID protocols and for PRNGs. SPONGENT-128 and SPONGENT-160 might be used in highly constrained applications with low and middle requirements for collision security. The latter also provides compatibility to the SHA-1 interfaces. The parameters of SPONGENT-224 and SPONGENT-256 correspond to those of a subset of SHA-2 and SHA-3 to make SPONGENT compatible to the standard interfaces in usual lightweight embedded scenarios.

\subsection{Organization of the article}

The remainder of the article is organized as follows. Section 2 describes the design of SPONGENT and gives a design rationale. Section 3 presents some results of security analysis, including proven lower bounds on the number of differentially active S-boxes, best differential characteristics found, rebound attacks, and linear attacks. In Section 4 , the implementation results are given for a range of trade-offs. We conclude in Section 5 .

\section{THE DESIGN OF SPONGENT}

SPONGENT is a sponge construction based on a wide PRESENT-type permutation. Given a finite number of input bits, it produces an $n$-bit hash value. A design goal for SPONGENT is to follow the hermetic sponge strategy (no structural distinguishers for the underlying permutation are allowed).

\subsection{Permutation-based sponge construction}

SPONGENT relies on a sponge construction - a simple iterated design that takes a variable-length input and can produce an output of an arbitrary length based on a permutation $\pi_{b}$ operating on a state of a fixed number $b$ of bits. The size of the internal state $b=r+c \geq n$ is called width, where $r$ is the rate and $c$ the capacity. 
The sponge construction proceeds in three phases (see also Figure 1):

- Initialization phase: the message is padded by a single bit 1 followed by a necessary number of 0 bits up to a multiple of $r$ bits (e.g., if $r=8$, then the 1-bit message ' 0 ' is transformed to '01000000'). Then it is cut into blocks of $r$ bits.

- Absorbing phase: the $r$-bit input message blocks are xored into the first $r$ bits of the state, interleaved with applications of the permutation $\pi_{b}$.

- Squeezing phase: the first $r$ bits of the state are returned as output, interleaved with applications of the permutation $\pi_{b}$, until $n$ bits are returned.

In SPONGENT, the $b$-bit 0 is taken as the initial value before the absorbing phase. In all SPONGENT variants, except SPONGENT-88/80/8, the hash size $n$ equals either capacity $c$ or $2 c$. The message chunks are xored into the $r$ rightmost bit positions of the state. The same $r$ bit positions form parts of the hash output.

Let a permutation-based sponge construction have $n \geq c$ and $c / 2>r$ which is fulfilled for the parameter choices of most of the SPONGENT variants. Then the works [2], [3], [9] imply the preimage security of $2^{n-r}$ as well as the second preimage and collision securities of $2^{c / 2}$ if this construction is hermetic (that is, if the underlying permutation does not have any structural distinguishers). The best preimage attack we are aware of in this case has a computational complexity of $2^{n-r}+2^{c / 2}$. Later, this work is extended in [14] and preimage security is defined more generalized form: $\min \left(2^{\min (n, c+r)}, \max \left(2^{\min (n-r, c)}, 2^{c / 2}\right)\right)$.

For permutation-based sponge constructions with $n<$ $c$ and $c / 2 \leq r$ such as the remaining SPONGENT variants, it follows from the same works that the second preimage security is $2^{n}$ and collision security is $2^{c / 2}$. The previous preimage attack also works for this case hence we claim that the preimage security is $\min \left(2^{n}, \max \left(2^{n-r}, 2^{c / 2}\right)\right)$ since $n-r<c$.

\subsection{Parameters}

We propose 13 variants of SPONGENT with five different hash output lengths at multiple security levels, see Table 1

\subsection{PRESENT-type permutation}

The permutation $\pi_{b}: \mathbb{F}_{2}^{b} \rightarrow \mathbb{F}_{2}^{b}$ is an $R$-round transform of the input STATE of $b$ bits that can be outlined at a top-level as:

$$
\begin{aligned}
& \text { for } i=1 \text { to } R \text { do } \\
& \text { STATE } \leftarrow \operatorname{rgfnINOJI}_{b}(i) \oplus \operatorname{STATE} \oplus \operatorname{lCounter}_{b}(i) \\
& \text { STATE } \leftarrow \text { sBoxLayer }_{b} \text { (STATE) } \\
& \text { STATE } \leftarrow \text { pLayer }_{b} \text { (STATE) } \\
& \text { end for }
\end{aligned}
$$

where sBoxLayer ${ }_{b}$ and pLayer $_{b}$ describe how the STATE evolves. For ease of design, only widths $b$ with $4 \mid b$ are allowed. The number $R$ of rounds depends on block size
TABLE 2

Initial values of ICounter ${ }_{b}$ for all SPONGENT variants.

\begin{tabular}{l|c|c}
\hline & LFSR size (bit) & Initial Value (hex) \\
\hline SPONGENT-88/80/8 & 6 & 05 \\
SPONGENT-88/176/88 & 8 & $\mathrm{C} 6$ \\
\hline SPONGENT-128/128/8 & 7 & $7 \mathrm{~A}$ \\
SPONGENT-128/256/128 & 8 & $\mathrm{FB}$ \\
\hline SPONGENT-160/160/16 & 7 & 45 \\
SPONGENT-160/160/80 & 7 & 01 \\
SPONGENT-160/320/160 & 8 & $\mathrm{A7}$ \\
\hline SPONGENT-224/224/16 & 7 & 01 \\
SPONGENT-224/224/112 & 8 & 52 \\
SPONGENT-224/448/224 & 9 & 105 \\
\hline SPONGENT-256/256/16 & 8 & $9 \mathrm{E}$ \\
SPONGENT-256/256/128 & 8 & $\mathrm{FB}$ \\
SPONGENT-256/512/256 & 9 & 015 \\
\hline
\end{tabular}

$b$ and can be found in Subsection 2.2 (see also Table 1 . lCounter $_{b}(i)$ is the state of an LFSR dependent on $b$ at time $i$ which yields the round constant in round $i$ and is added to the rightmost bits of STATE. $\operatorname{rgfnumOJI}_{b}(i)$ is the value of $1_{\text {Counter }}(i)$ with its bits in reversed order and is added to the leftmost bits of STATE.

The following building blocks are generalizations of the PRESENT structure to larger $b$-bit widths:

1) sBoxLayer $_{b}$ : This denotes the use of a 4-bit to 4bit S-box $S: \mathbb{F}_{2}^{4} \rightarrow \mathbb{F}_{2}^{4}$ which is applied $b / 4$ times in parallel. The action of the S-box in hexadecimal notation is given by the following table:

\begin{tabular}{c|cccccccccccccccc}
\hline$x$ & 0 & 1 & 2 & 3 & 4 & 5 & 6 & 7 & 8 & 9 & A & B & C & D & E & F \\
\hline$S[x]$ & E & D & B & 0 & 2 & 1 & 4 & F & 7 & A & 8 & 5 & 9 & C & 3 & 6 \\
\hline
\end{tabular}

2) pLayer $_{b}$ : This is an extension of the (inverse) PRESENT bit-permutation and moves bit $j$ of STATE to bit position $P_{b}(j)$, where

$P_{b}(j)= \begin{cases}j \cdot b / 4 \quad \bmod b-1, & \text { if } j \in\{0, \ldots, b-2\} \\ b-1, & \text { if } j=b-1 .\end{cases}$

and can be seen in Figure 2

3) 1 Counter ${ }_{b}$ : This is one of the four $\left\lceil\log _{2} R\right\rceil$-bit LFSRs. The LFSR is clocked once every time its state has been used and its final value is all ones. If $\zeta$ is the root of unity in the corresponding binary finite field, the $n$-bit LFRSs defined by the polynomials given below are used for the SPONGENT variants.

\begin{tabular}{c|l}
\hline LFSR size (bit) & Primitive Polynomial \\
\hline 6 & $\zeta^{6}+\zeta^{5}+1$ \\
7 & $\zeta^{7}+\zeta^{6}+1$ \\
8 & $\zeta^{8}+\zeta^{4}+\zeta^{3}+\zeta^{2}+1$ \\
9 & $\zeta^{9}+\zeta^{4}+1$ \\
\hline
\end{tabular}

Table 2 provides sizes and initial values of all the LFSRs.

\subsection{Design rationale}

The overall design approach for SPONGENT is to target low area while favoring simplicity. 
TABLE 1

13 SPONGENT variants.

\begin{tabular}{l|c|ccccc|ccc}
\hline & $\begin{array}{c}n \\
\text { (bit) }\end{array}$ & $\begin{array}{c}b \\
\text { (bit) }\end{array}$ & $\begin{array}{c}c \\
\text { (bit) }\end{array}$ & $\begin{array}{c}r \\
\text { (bit) }\end{array}$ & $\begin{array}{c}R \text { number } \\
\text { of rounds }\end{array}$ & pre. & $\begin{array}{c}\text { security(bit) } \\
\text { 2nd pre. }\end{array}$ & col. \\
\hline SPONGENT-88/80/8 & 88 & 88 & 80 & 8 & 45 & 80 & 40 & 40 \\
SPONGENT-88/176/88 & 88 & 264 & 176 & 88 & 135 & 88 & 88 & 44 \\
\hline SPONGENT-128/128/8 & 128 & 136 & 128 & 8 & 70 & 120 & 64 & 64 \\
SPONGENT-128/256/128 & 128 & 384 & 256 & 128 & 195 & 128 & 128 & 64 \\
\hline SPONGENT-160/160/16 & 160 & 176 & 160 & 16 & 90 & 144 & 80 & 80 \\
SPONGENT-160/160/80 & 160 & 240 & 160 & 80 & 120 & 80 & 80 & 80 \\
SPONGENT-160/320/160 & 160 & 480 & 320 & 160 & 240 & 160 & 160 & 80 \\
\hline SPONGENT-224/224/16 & 224 & 240 & 224 & 16 & 120 & 208 & 112 & 112 \\
SPONGENT-224/224/112 & 224 & 336 & 224 & 112 & 170 & 112 & 112 & 112 \\
SPONGENT-224/448/224 & 224 & 672 & 448 & 224 & 340 & 224 & 224 & 112 \\
\hline SPONGENT-256/256/16 & 256 & 272 & 256 & 16 & 140 & 240 & 128 & 128 \\
SPONGENT-256/256/128 & 256 & 384 & 256 & 128 & 195 & 128 & 128 & 128 \\
SPONGENT-256/512/256 & 256 & 768 & 512 & 256 & 385 & 256 & 256 & 128 \\
\hline
\end{tabular}

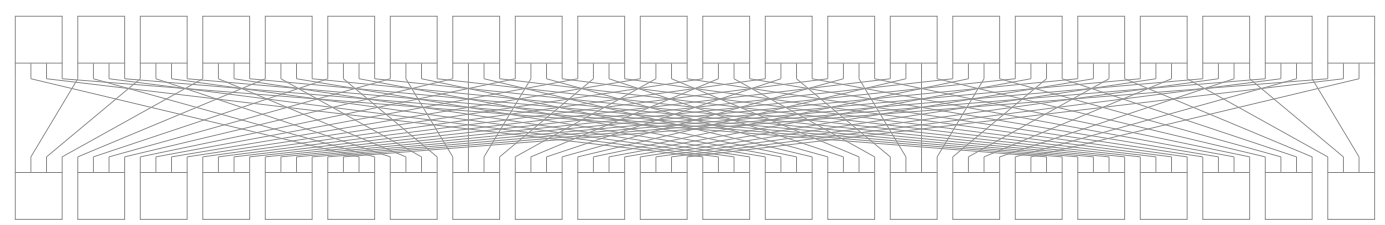

Fig. 2. The bit permutation layer of SPONGENT-88 at the example of pLayer $_{88}$.

The 4-bit S-box is the major block of functional logic in a serial low-area implementation of SPONGENT. It fulfills the PRESENT design criteria in terms of differential and linear properties [5]. Moreover, any linear approximation over the S-box involving only single bits both in the input and output masks is unbiased. This aims to restrict the linear hull effect discovered in round-reduced PRESENT.

The function of the bit permutation pLayer is to provide good diffusion, by acting together with the S-box, while having a limited impact on the area requirements. This is its main design goal, while a bit permutation may occupy additional space in silicon. The counters lCounter and rofnuwoJl are mainly aimed to prevent sliding properties and make prospective cryptanalysis approaches using properties like invariant subspaces [20] more involving.

The structures of the bit permutation and the S-box in SPONGENT make it possible to prove the following differential property (see Subsection 3.1 for the proof):

Theorem 1. Any 5-round differential characteristic of the underlying permutation of SPONGENT with $b \geq 64$ has a minimum of 10 active S-boxes. Moreover, any 6-round differential characteristic of the underlying permutation of SPONGENT with $b \geq 256$ has a minimum of 14 active Sboxes.

The concept of counting active S-boxes is central to the differential cryptanalysis. The minimum number of active S-boxes relates to the maximum differential characteristic probability of the construction. Since in the hash setting there are no random and independent key values added between the rounds, this relation is not exact (in fact that it is even not exact for most practical keyed block ciphers). However, differentially active Sboxes are still the major technique used to evaluate the security of SPN-based hash functions.

An important property of the SPONGENT S-box is that its maximum differential probability is $2^{-2}$. This fact and the assumption of the independency of difference propagation in different rounds yield an upper bound on the differential characteristic probability of $2^{-20}$ over 5 rounds and of $2^{-28}$ over 6 rounds for $b \geq 256$ which follows from the claims of Theorem 1

Theorem 1 is used to determine the number $R$ of rounds in permutation $\pi_{b}: R$ is chosen in a way that $\pi_{b}$ provides at least $b$ active S-boxes. Other types of analysis are performed in the next section.

\section{Security Analysis}

In this section, we discuss the security of SPONGENT against known cryptanalytic attacks by applying the most important state-of-the-art methods of cryptanalysis and investigating their complexity.

\subsection{Resistance against differential cryptanalysis}

Here we analyze the resistance of SPONGENT against differential attacks where Theorem 1 plays a key role providing a lower bound on the number of active Sboxes in a differential characteristic. The similarities of the SPONGENT permutations and the basic PRESENT 
TABLE 3

Differential characteristics with lowest numbers of differentially active S-boxes (ASN). The probabilities are calculated assuming the independency of round computations.

\begin{tabular}{|c|c|c|c|c|c|c|c|c|c|c|}
\hline \multirow{2}{*}{$\begin{array}{l}\text { \# of } \\
\text { rounds }\end{array}$} & \multicolumn{2}{|c|}{ SPONGENT-88/80/8 } & \multicolumn{2}{|c|}{ SPONGENT-128/128/8 } & \multicolumn{2}{|c|}{ SPONGENT-160/160/16 } & \multicolumn{2}{|c|}{ SPONGENT-224/224/16 } & \multicolumn{2}{|c|}{ SPONGENT-160/160/80 } \\
\hline & ASN & Prob & ASN & Prob & ASN & Prob & ASN & Prob & ASN & Prob \\
\hline 5 & 10 & $2^{-21}$ & 10 & $2^{-22}$ & 10 & $2^{-21}$ & 10 & $2^{-21}$ & 14 & $2^{-21}$ \\
\hline 10 & 20 & $2^{-47}$ & 24 & $2^{-60}$ & 20 & $2^{-50}$ & 20 & $2^{-43}$ & 32 & $2^{-43}$ \\
\hline 15 & 30 & $2^{-74}$ & 40 & $2^{-101}$ & 30 & $2^{-79}$ & 30 & $2^{-66}$ & 52 & $2^{-66}$ \\
\hline \multirow{2}{*}{$\begin{array}{l}\# \text { of } \\
\text { rounds }\end{array}$} & \multicolumn{2}{|c|}{ SPONGENT- $88 / 176 / 88$} & \multicolumn{2}{|c|}{ SPONGENT-128/256/128 } & \multicolumn{2}{|c|}{ SPONGENT-160/320/160 } & \multicolumn{2}{|c|}{ SPONGENT-224/224/112 } & \multicolumn{2}{|c|}{ SPONGENT-24/448/224 } \\
\hline & ASN & Prob & ASN & Prob & ASN & Prob & ASN & Prob & ASN & Prob \\
\hline 6 & 14 & $2^{-28}$ & 14 & $2^{-28}$ & 14 & $2^{-28}$ & 14 & $2^{-28}$ & 14 & $2^{-28}$ \\
\hline 12 & 41 & $2^{-96}$ & 37 & $2^{-72}$ & 39 & $2^{-93}$ & 36 & $2^{-88}$ & & \\
\hline 18 & 64 & $2^{-158}$ & 52 & $2^{-119}$ & 65 & $2^{-157}$ & 66 & $2^{-174}$ & & \\
\hline \multirow{2}{*}{$\begin{array}{l}\# \text { of } \\
\text { rounds }\end{array}$} & SPON & $256 / 256 / 16$ & \multicolumn{2}{|c|}{ SPONGENT-256/256/128 } & \multicolumn{2}{|c|}{ SPONGENT-256/512/256 } & & & & \\
\hline & ASN & Prob & ASN & Prob & ASN & Prob & & & & \\
\hline 6 & 14 & $2^{-28}$ & 14 & $2^{-28}$ & 14 & $2^{-28}$ & & & & \\
\hline 12 & 32 & $2^{-73}$ & 50 & $2^{-123}$ & 34 & $2^{-84}$ & & & & \\
\hline 18 & 52 & $2^{-128}$ & 68 & $2^{-169}$ & 54 & $2^{-128}$ & & & & \\
\hline
\end{tabular}

cipher allow to reuse some of the results obtained for PRESENT in [5]. More precisely, the results on the number of differentially active S-boxes over 5 and 6 rounds will hold for all respective SPONGENT variants which is reflected in Theorem 1 1 . The proof of the Theorem 1 is as follows:

Proof: [Theorem 1] The statements for SPONGENT variants with $64 \leq b \leq 255$ can directly be proven by applying the same technique used in [5, Appendix III]. The proof of the 6-round bounds for SPONGENT variants with $b \geq 256$ in Theorem 1 is based on some extended observations. Here, we will only give the proof for when the width, $b$, is a multiple of 64 bits, i.e., $b=64 n$. The proof for other $b$ values can also be obtained by making use of the observations given below. Since the proof is specific to each $b$ and hence more tedious, we do not present them here.

We obtain $n$ groups and $4 n$ subgroups by calling each four consecutive S-boxes as a subgroup and each sixteen consecutive S-boxes as a group. To be more specific: subgroup $i$ is comprised of the S-boxes $[4(i-1) \ldots 4 i-1]$ and similarly group $j$ has the subgroups $[4(j-1) \ldots 4 j-1]$. (see Figure 3). By examining the substitution and linear layers, one can make the following observations:

1) The S-box of SPONGENT is such that a difference in single input bit causes a difference in at least two output bits or vice versa.

2) The input bits to an S-box come from four distinct S-boxes of the same subgroup.

3) The input bits to a subgroup of four S-boxes come from 16 distinct S-boxes of the same group.

4) The input bits to a group of 16 S-boxes come from 64 different S-boxes.

5) The four output bits from a particular S-box enter four distinct S-boxes, each of which belongs to a distinct group of S-boxes in the subsequent round.

6) The output bits of S-boxes in distinct groups go to distinct S-boxes in distinct subgroups.

7) The output bits of S-boxes in distinct subgroups go to distinct S-boxes.

For the latter statement (SPONGENT-256), one has to deal with more cases. Consider six consecutive rounds of SPONGENT ranging from $i$ to $i+5$ for $i \in[1 \ldots 155]$. Let $D_{j}$ be the number of active S-boxes in round $j$. If $D_{j} \geq 3$, for $i \leq j \leq i+5$, then the theorem trivially holds. So let us suppose that one of $D_{j}$ is equal to one first and to two then. We have the following cases:

Case $D_{i+2}=1$. By using observation 1 , we can deduce that $D_{i+1}+D_{i+3} \geq 3$ and all active S-boxes of round $i+1$ belong to the same subgroup from observation 2. Each of these active S-boxes have only a single bit difference in their output. So, according to observation 3 we have that $D_{i} \geq 2 D_{i+1}$. Conversely, according to observation 5 , all active S-boxes in round $i+3$ belong to distinct groups and have only a single bit difference in their input. So, according to observation 6 , we have that $D_{i+4} \geq 2 D_{i+3}$. Moreover, all active S-boxes in round $i+4$ belong to distinct subgroups and have only a single bit difference in their input. Thus, by using observation 7 , we obtain that $D_{i+5} \geq 2 D_{i+4}$ and can conclude that $\sum_{j=i}^{i+5} D_{j} \geq$ $1+3+2 \times 3+4 D_{i+3} \geq 14$.

Case $D_{i+3}=1 \quad$ If $D_{i+2}=1$ we can refer to the first case. So, suppose that $D_{i+2} \geq 2$. According to the observation 2 , all active $\mathrm{S}$-boxes of round $i+2$ belong to the same subgroup and each of these active S-boxes has only a single bit difference in their output. Thus, according to observation $3, D_{i+1} \geq 2 D_{i+2} \geq 4$. Since all active S-boxes in round $i+1$ belong to distinct S-boxes of the same group and have only a single bit difference in their input, according to observation 4 , we have that $D_{i} \geq 2 D_{i+1}$. On the opposite, $D_{i+4}$ and $D_{i+5}$ can get one and two as a minimum value, respectively. Together this gives $\sum_{j=i}^{i+5} D_{j} \geq 8+4+2+1+1+2 \geq 18$.

Case $D_{i+1}=1$ If $D_{i+2}=1$, then we can refer to the first case. Thus, suppose that $D_{i+2} \geq 2$. According to observation 5 , all active S-boxes in round $i+2$ belong to 


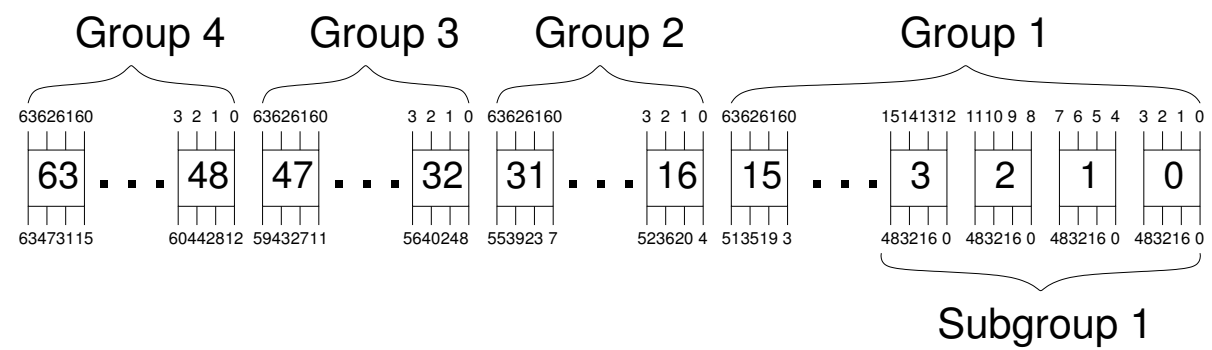

Fig. 3. The grouping and subgrouping of S-boxes for $b=256$. The input numbers indicate the S-box origin from the previous round and the output numbers indicate the destination S-box in the following round.

distinct groups and have only a single bit difference in their input. Thus, according to observation 6 , we have that $D_{i+3} \geq 2 D_{i+2}$. Since all active S-boxes in round $i+3$ belong to distinct subgroups and have only a single bit difference in their input. Therefore, according to observation 7 , we have that $D_{i+4} \geq 2 D_{i+3}$. To sum up, $\sum_{j=i}^{i+5} D_{j} \geq 1+1+2+4+8+D_{i+5} \geq 16+D_{i+5} \geq 17$, since $D_{i+4}>0$ implies that $D_{i+5} \geq 1$.

Case $D_{i+4}=1$ If $D_{i+3}=1$, then we can refer to the second case. So, suppose that $D_{i+3} \geq 2$. According to the observation 2, all active S-boxes of round $i+3$ belong to the same subgroup and each of those active S-boxes has only a single bit difference in their output. Therefore, according to observation 3 , we have that $D_{i+2} \geq D_{i+3}$. Since, all active S-boxes in round $i+2$ belong to distinct S-boxes of the same group and have only a single bit difference in their input, according to observation 4 , we have that $D_{i+1} \geq 2 D_{i+2}$. Since $D_{i+1}>0, D_{i} \geq 1$. Thus, we can conclude that $\sum_{j=i}^{i+5} D_{j} \geq D_{i}+8+4+2+1+1 \geq$ $D_{i}+16 \geq 17$.

Cases $D_{i}=1$ and $D_{i+5}=1$ are similar to the those for the third and fourth cases.

So far we have considered all paths including one active S-box in one of the rounds and obtained 14 as the minimum number of active S-boxes. But if there exists a path that has two active S-boxes in each round, then the lower bound would be 12 . For this purpose, without loss of generality, assume:

$D_{i+1}=D_{i+2}=D_{i+3}=2 \quad$ The two active S-boxes in $i+2$ are either in the same subgroup or in different subgroups. For the former, from observations 3 and 7 , we know that they have single bit of differences coming from two different subgroups of the same group in round $i+1$. From observation 1 , these two S-boxes have at least two bits of input difference, hence we obtain $D_{i}=4$ by observation 2 and 3 . Furthermore the two Sboxes in round $i+2$ have two bits of output difference by observation 1 . Hence, in round $i+3$, the active Sboxes have two bits of input and they are in distinct groups by observation 5 . Therefore, it is possible to have $D_{i+4}=2$ in distinct subgroups. Hence by using observation 7, we obtain $D_{i+5}=4$. Thus, we can conclude that $\sum_{j=i}^{i+5} D_{j} \geq 4+2+2+2+2+4 \geq 16$.

For the latter, the two active S-boxes in round $i+1$ must
TABLE 4

Longest differential characteristics holding with probability in the range of $2^{-b}$ (under independency assumption)

\begin{tabular}{l|ccl}
\hline & \# rounds & ASN & Prob \\
\hline SPONGENT-88/80/8 & 17 & 34 & $2^{-88}$ \\
SPONGENT-88/176/88 & 27 & 103 & $2^{-268}$ \\
\hline SPONGENT-128/128/8 & 20 & 56 & $2^{-137}$ \\
SPONGENT-128/256/128 & 42 & 146 & $2^{-385}$ \\
\hline SPONGENT-160/160/16 & 20 & 66 & $2^{-179}$ \\
SPONGENT-160/160/80 & 44 & 88 & $2^{-242}$ \\
SPONGENT-160/320/160 & 48 & 192 & $2^{-480}$ \\
\hline SPONGENT-224/224/16 & 44 & 88 & $2^{-242}$ \\
SPONGENT-224/224/112 & 26 & 133 & $2^{-343}$ \\
SPONGENT-224/448/224 & - & - & - \\
\hline SPONGENT-256/256/16 & 30 & 108 & $2^{-276}$ \\
SPONGENT-256/256/128 & 31 & 150 & $2^{-392}$ \\
SPONGENT-256/512/256 & 85 & 256 & $2^{-768}$ \\
\hline
\end{tabular}

have two bits of input and by observation 2 their input bits should be coming from distinct S-boxes in the same subgroup. So, the problem is reduced to the former case with one round of shift, and we can immediately say that $D_{i}=2$ and $D_{i+4}=4$. Hence by using observation 7 , we obtain $D_{i+5}=4$. Thus, we can conclude that $\sum_{j=i}^{i+5} D_{j} \geq$ $2+2+2+2+4+4 \geq 16$.

Based on these results, we conclude that the longest run with two active S-boxes in each round is four rounds, and the number of active S-boxes cannot be less than 14 .

For all SPONGENT variants, we found that those 5and 6-round bounds are actually tight. We present the characteristics attaining them in Table 3 . Additionally, we perform a branch-and-bound search for longest characteristics with probabilities in the range of $2^{-b}$. The results are given in Table 4. most of them based in iterative characteristics.

\subsection{Collision attacks}

A natural approach to obtain a collision for a sponge construction is to inject a difference in a message block and then cancel the propagated difference by a difference in the next message block, i.e., $\left(0 \ldots 0 \| \Delta m_{i}\right) \stackrel{\pi}{\rightarrow}$ $\left(0 \ldots 0 \| \Delta m_{i+1}\right)$. For this purpose, we follow a narrow 
trail strategy using truncated differential characteristics. We start from a given input difference (some difference restricted to S-boxes that the message block is xored into) and look for all paths that go to a fixed output difference (also located in the bitrate part of the state). Based on our experiments, even by using truncated differential characteristics, the probability of such a path is quite low and it is not possible to attack the full number of rounds.

\subsubsection{Rebound attack}

The rebound attack [21], a recent technique for cryptanalysis of hash functions, is applicable to both block cipher based and permutation based hash constructions. It consists of two main steps: the inbound phase where the freedom is used to connect the middle rounds by using the match-in-the-middle technique and the outbound phase where the connected truncated differentials are calculated in both forward and backward directions. It has been mostly used to improve the results on AES-based algorithms (e.g. Grøstl [13]), but it has also been successfully applied to similar permutations (e.g. Keccak [12]).

Compared to the other algorithms the rebound attack has been successfully applied to, the design of SPONGENT imposes some limitations. First of all, since the permutation is bit-oriented, and not byte-oriented, it might be non-trivial to find the path followed by a given input difference and to determine the number of active S-boxes after several rounds. This is mainly due to the difference propagation that strongly depends on the values of the passive part of the state. Moreover, the probability that two inbound phases match requires more detailed analysis. Below we attempt to develop rebound attacks on several SPONGENT variants. Rebound analysis applies similarly to the remaining variants.

For SPONGENT-88/80/8, we looked for characteristics that match in the middle with the available degrees of freedom coming from the message bits. For 5 and more rounds, when the whole state is active in the matching phase, we would not be able to generate enough pairs by using only a difference in the message bits. Since the expected probability of matching the inbound phases is $2^{-b / 4}$ (where $b / 4$ is the number of S-boxes) and the available degree of freedom is only $2^{2 r}$, this argument is also valid for SPONGENT-128/128/8, SPONGENT-160/160/16, SPONGENT-224/224/16, and SPONGENT-256/256/16. For other SPONGENT variants there exist enough degrees of freedom and we decided to explore it with one of the SPONGENT variants.

It is trivial to find one round inbound phase in SPONGENT and then by applying the outbound phase for several rounds, which technically yields a differential characteristic. Since, one third of the state is xored with the message value for the variants whose rate is different from 8 or 16 , we have enough flexibility to diffuse the difference through forward and backward direction. But then, merging these differential characteristics seems

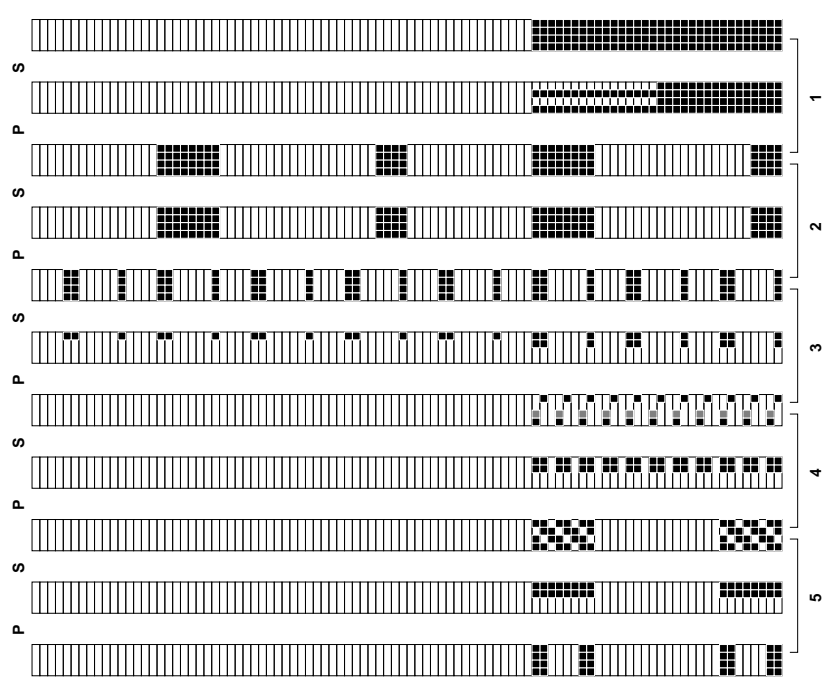

Fig. 4. Differential path for the rebound attack on SPONGENT-128/256/128 (S: sBoxLayer ${ }_{384}$, P: pLayer $_{384}$ ).

difficult due to the limited number of pairs generated in the inbound phase.

In our example which is given in Figure 4 , we focused on SPONGENT-128/256/128 and found a five-round trail by following the strategy outlined above. In our attack, we fix the input and output differences of sBoxLayer in the fourth round. For a half of the differences, we fix the difference to $1_{x} \rightarrow 3_{x}$ and for the other half it is possible to fix the difference to either $4_{x} \rightarrow 3_{x}$ or $8_{x} \rightarrow 3_{x}$, but not both together. Then, we let the differences diffuse for three rounds in the backward direction and for one round in the forward direction. All possible positions of the active bits are shown in black in Figure 4 Note that in round 5, we impose a restriction on the outputs of the SBoxLayer such that the differences occur only in the bitrate part.

It is possible to generate $4^{11} \cdot 2^{11}=2^{33}$ pairs in the inbound phase and a pair can satisfy the desired differential trail with a probability of $\operatorname{Pr}\left[B_{x} \rightarrow\left\{1_{x}, 2_{x}\right\}\right]^{6}$. $\operatorname{Pr}\left[D_{x} \rightarrow\left\{1_{x}, 2_{x}, 3_{x}\right\}\right]^{6} \cdot \operatorname{Pr}\left[6_{x} \rightarrow\left\{1_{x}, 2_{x}\right\}\right]^{4}=2^{-26.15}$. Therefore, in total, we expect to have $2^{6.85}$ valid pairs that satisfy the given path.

\subsubsection{Bound considerations for the rebound attack}

The adversary might try to find a way to attack by using multiple inbounds with a sparse differential. Therefore, to explore the security against multiple inbound phases, we put the adversary into a best-case scenario as follows.

We know that there exists no differential characteristic over five rounds with the number of active S-boxes less than 10 for all SPONGENT variants. We can also deduce lower bounds on the number of active S-boxes for 1,2,3, and 4 rounds as 1,2,4 and 6, respectively. Then a bound on the minimum number of active S-boxes, hence the probability of a differential characteristic, for any number of rounds can be approximated by combining these bounds $\sqrt{1}$

1. Note that, Table 3 shows that these bounds might be optimistic. 
TABLE 5

Bounds for rebound attack.

\begin{tabular}{l|cc|cc}
\hline & \multicolumn{2}{|c|}{ 2 Inbounds } & \multicolumn{2}{c}{ 3 Inbounds } \\
& $\begin{array}{c}\text { rounds } \\
\text { /inbound }\end{array}$ & $\begin{array}{c}\text { attacked } \\
\text { rounds(\%) }\end{array}$ & \multicolumn{2}{c}{ rounds } \\
/inbound & $\begin{array}{c}\text { attacked } \\
\text { rounds(\%) }\end{array}$ \\
\hline SPONGENT-88/80/8 & 9 & 40.00 & 9 & 60.00 \\
SPONGENT-88/176/88 & 10 & 14.81 & 9 & 20.00 \\
\hline SPONGENT-128/128/8 & 15 & 42.86 & 14 & 60.00 \\
SPONGENT-128/256/128 & 14 & 14.36 & 13 & 20.00 \\
\hline SPONGENT-160/160/16 & 19 & 42.22 & 19 & 63.33 \\
SPONGENT-160/160/80 & 19 & 31.67 & 19 & 47.50 \\
SPONGENT-160/320/160 & 17 & 14.17 & 16 & 20.00 \\
\hline SPONGENT-224/224/16 & 28 & 46.67 & 27 & 67.50 \\
SPONGENT-224/224/112 & 23 & 27.06 & 23 & 40.59 \\
SPONGENT-224/448/224 & 23 & 13.53 & 23 & 20.29 \\
\hline SPONGENT-256/256/16 & 28 & 40.00 & 27 & 57.86 \\
SPONGENT-256/256/128 & 28 & 28.72 & 27 & 41.54 \\
SPONGENT-256/512/256 & 28 & 14.55 & 27 & 21.04 \\
\hline
\end{tabular}

The desired bit security level for a sponge construction with respect to collision attacks is $c / 2$. From now on we assume that the complexity of each inbound phase is equal to $c / 2$ and at least one active S-box matches between two inbound phases (with probability $2^{-8}$ ). Let $n_{\text {in }}$ be the number of inbound phases then we have to generate $n_{\text {elm }}=2^{8 \cdot\left(n_{i n}-1\right) / n_{\text {in }}}$ elements for each inbound phase. Let $p$ denote the probability of each inbound phase, then $p$ can be at least $2^{-\left(c / 2-\left\lceil\log _{2}\left(n_{e l m}\right)\right\rceil\right)}$ and we can compute the number of rounds in each inbound phase by using the given bounds above.

Under these assumptions, the maximum number of rounds per inbound phase and the percentage of the total number of rounds attacked is given in Table 5 .

\subsection{Preimage resistance}

Here we apply a meet-in-the-middle approach to obtain preimages on SPONGENT. The attack has two main steps: pre-computation and matching phase. Complexity of the attack is dominated by pre-computation phase.

Since the hash size is $n$ bits, and the data is extracted in $r$ bit chunks, there exists $n / r$ rounds in the squeezing phase. To be able to compute the data backwards in the absorbing phase, we need to know not only $h_{i}$ 's but also $d_{i}$ values to obtain the input value of the permutation $\pi$, where $h_{i}$ denotes the part of the hash value and $d_{i}$ is the concatenated part to $h_{i}$. The algorithm is as follows:

1) Pre-computation: We know that $\pi^{-1}\left(h_{i+1}, d_{i+1}\right)=$ $\left(h_{i}, d_{i}\right)$ for each $i$ in the squeezing phase. Since $h_{i}$ (r-bits) is already fixed, the probability of finding such $d_{i}$ is $2^{-r}$. Therefore, we start with $2^{((n / r)-1) \cdot r}=2^{n-r}$ different $d_{n / r}$ values to have a solution for $d_{1}$.

2) Match-in-the-middle: Choose $k$ such that $k \cdot r \geq$ $c / 2$. Then

- Generate $2^{c / 2}$ elements in the backward direction by using $\left(h_{1}, d_{1}\right)$ and possible values for $m_{k+2}, \ldots, m_{2 k+1}$ and store them in a table.

- Generate $2^{c / 2}$ elements in the forward direction by using possible values for $m_{1}, \ldots, m_{k}$ and
TABLE 6

Meet-in-the-middle attack results for SPONGENT.

\begin{tabular}{l|rrr}
\hline & $\begin{array}{r}\text { Time Complexity } \\
\max \left(2^{n-r}, 2^{c / 2}\right)\end{array}$ & $\begin{array}{r}\text { Memory Complexity } \\
\left(2^{c / 2}\right)\end{array}$ \\
\hline SPONGENT-88/80/8 & 5 & $2^{80}$ & $2^{40}$ \\
SPONGENT-88/176/88 & 1 & $2^{88}$ & $2^{88}$ \\
\hline SPONGENT-128/128/8 & 8 & $2^{120}$ & $2^{64}$ \\
SPONGENT-128/256/128 & 1 & $2^{128}$ & $2^{80}$ \\
\hline SPONGENT-160/160/16 & 5 & $2^{144}$ & $2^{80}$ \\
SPONGENT-160/160/80 & 1 & $2^{80}$ & $2^{160}$ \\
SPONGENT-160/320/160 & 1 & $2^{160}$ & $2^{112}$ \\
\hline SPONGENT-224/224/16 & 7 & $2^{208}$ & $2^{112}$ \\
SPONGENT-224/224/112 & 1 & $2^{112}$ & $2^{224}$ \\
SPONGENT-224/448/224 & 1 & $2^{224}$ & $2^{128}$ \\
\hline SPONGENT-256/256/16 & 8 & $2^{240}$ & $2^{128}$ \\
SPONGENT-256/256/128 & 1 & $2^{128}$ & $2^{256}$ \\
SPONGENT-256/512/256 & 1 & $2^{256}$ &
\end{tabular}

compare with list in the previous step to find a match of $c$ bits (corresponding to capacity) in the middle.

- Obtain $m_{k+1}$ by xor-ing the $r$ bits (corresponding to bitrate) for the matching elements.

In the pre-computation part, we obtain the required value $d_{1}$ to compute the data backwards in the absorbing phase by $2^{n-r}$ computations. We need $2^{c / 2}$ memory to store the elements generated in the second step and $2^{c / 2}$ computations are needed to find a full match. These complexities are exactly given in [25] which extends the bounds given in [9] for $c>n$. We have derived those once again here for completeness. The preimage attack complexities together with the parameter $k$ are given in Table 6

Note that, if $c \leq n-r$, it is sufficient to try all possible $2^{c}$ values to construct the whole state in order to obtain a preimage, hence it provides an upper bound for the preimage resistance. If we combine the results we obtain $\left.\max \left(2^{\min (n-r, c}\right), 2^{c / 2}\right)$ and it can be generalized into the form: $\min \left(2^{\min (n, c+r)}, \max \left(2^{\min (n-r, c)}, 2^{c / 2}\right)\right)$. Here, $2^{\min (n, c+r)}$ computations will be necessary depending on the permutation size when the generic attack, defined above, fails.

\subsection{Linear attacks}

The most successful attacks, the attacks that can break the highest number of rounds, for the block cipher PRESENT are those based on linear approximations. In particular the multi-dimensional linear attack [7] and the statistical saturation attack [8] claim to break up to 26 rounds. It was shown in [19] that both attacks are closely related. Moreover, the main reason why these attacks are the most successful attacks on PRESENT so far, is the existence of many linear trails with only one active S-box in each round. It is not immediately clear how linear distinguishers on the SPONGENT permutation $\pi_{b}$ could be transferred into collision or (second) preimage attacks on the hash function. However, as we 


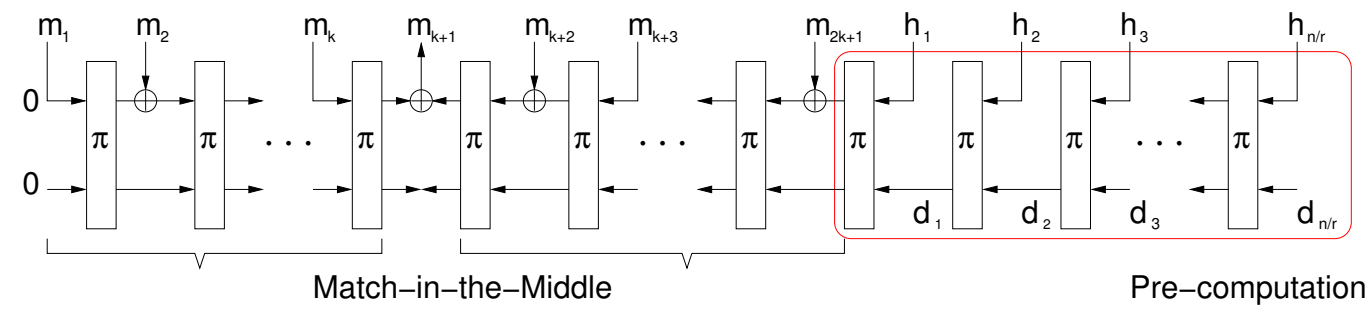

Fig. 5. Meet-in-the-middle attack against sponge construction.

claim that SPONGENT is a hermetic sponge construction, the existence of such distinguishers has to be excluded. So the SPONGENT S-box was chosen in a way that allows for at most one trail with this property given a linear approximation.

Unlike for the block cipher PRESENT, where the key determines the actual linear correlation between an input and an output mask, for the permutation $\pi_{b}$ we can compute the actual linear trail contribution for all trails with only one active S-box in every round. Each such trail over $w$ rounds has a correlation of $\pm 2^{-2 w}$ and for each trail determining the sign is easy. More concretely, one can easily compute a $b \times b$ matrix $M_{t}$ over the rationals such that the entry at position $i, j$ is the correlation coefficient for round $t$ for the linear trail with input mask $e_{i}$ and output mask $e_{j}$. Here $e_{i}$ (resp. $e_{j}$ ) is the unit vector with a single 1 at position $i$ (resp. $j$ ). Note that the matrices $M_{t}$ are sparse and all very similar, the only difference is caused by the round constant, which induces sign changes at a few positions only.

Given those matrices, it is now possible to compute the maximal linear correlation contribution for those one bit intermediate masks for all one bit input and output masks. For $w$ rounds we simply compute $M^{(w)}=\prod_{i=1}^{w} M_{i}$ and the maximal correlation is given by $c_{w}:=\max _{i, j}\left|M_{i j}^{(w)}\right|$. We compute this value for all SPONGENT variants. Table 7 summarizes those results. Most importantly, this table shows the maximal number of rounds $w$ where the trail contributions is still larger than or equal to $2^{-b / 2}$. Beyond this number of rounds, it seems unlikely that distinguishers based on linear approximations exist. For most SPONGENT variants, the best linear hull based on single-bit masks has exactly one linear trail.

\section{HARDWARE IMPLEMENTATION}

In this section we provide a wide range of hardware figures by evaluating all of the 13 SPONGENT variants in detail. Not only a comprehensive hardware evaluation is of our primary interest, we also further elaborate on the importance of having a unified benchmarking platform for comparing different lightweight designs. We therefore provide hardware figures of SPONGENT as well as two state-of-the-art lightweight hash functions (PHOTON and QUARK), all implemented using an open
TABLE 7

Results of linear trail correlation based on one bit masks.

\begin{tabular}{l|c|c|c|l}
\hline & $b$ & $\begin{array}{c}\max w \text { with } \\
c_{w} \geq 2^{-b / 2}\end{array}$ & $R$ & $\log _{2} c_{R}$ \\
\hline SPONGENT-88/80/8 & 88 & 22 & 45 & -90 \\
SPONGENT-88/176/88 & 264 & 66 & 135 & -270 \\
\hline SPONGENT-128/128/8 & 136 & 34 & 70 & -140 \\
SPONGENT-128/256/128 & 384 & 96 & 195 & -388.4 \\
\hline SPONGENT-160/160/16 & 176 & 44 & 90 & -180 \\
SPONGENT-160/160/80 & 240 & 60 & 120 & -240 \\
SPONGENT-160/320/160 & 480 & 122 & 240 & -473.7 \\
\hline SPONGENT-224/224/16 & 240 & 60 & 120 & -240 \\
SPONGENT-224/224/112 & 336 & 84 & 170 & -340 \\
SPONGENT-224/448/224 & 673 & 169 & 340 & -675.3 \\
\hline SPONGENT-256/256/16 & 272 & 68 & 140 & -280 \\
SPONGENT-256/256/128 & 384 & 96 & 195 & -388.4 \\
SPONGENT-256/512/256 & 768 & 192 & 385 & -770 \\
\hline
\end{tabular}

core $45 \mathrm{~nm}$ library (NANGATE2] [22]. To further stress on the latter issue, we present the performance of SPONGENT using four different CMOS technologies. As it will become apparent, the influence of a library choice on the hardware performance of a single design turns out to be paramount. This phenomenon, in the context of the SHA-3 competition, has been recently discussed in the literature [15].

A fair comparison of hardware performance between different designs has not been sufficiently addressed in the recent publications on lightweight cryptography. It is rather obvious that such comparison is only possible once the highly optimized designs are implemented on the same hardware platform, using the same standard cell library and the same synthesis tool (including the design flow scripts). In order to eliminate the influence of a specific implementation setup, the same experiment should ideally be repeated over many different instances of tools and libraries. Mainly due to the licensing issues and the designer's preference to use a certain software package this, however, becomes a very difficult task in practice.

To partially address this issue, we provide Table 8 where the design of SPONGENT is compared to state-ofthe-art lightweight hash functions QUARK and PHOTON, for which the RTL codes are publicly available. The comparison is done using NANGATE45, a publicly available

2. We use PDKv1_3_v2009_07 version with the typical case conditions NangateOpenCellLibrary_typical_conditional_ccs.lib 
open core library, while the synthesis is carried out using the same script and the same Cadence RTL compiler version 10.10-p104

What can be seen by observing the obtained data is that for the similar security levels (i.e. SPONGENT$88 / 80 / 8$ vs PHOTON-80/20/16, SPONGENT- $128 / 128 / 8$ vs U-QUARK vs PHOTON-128/16/16, SPONGENT$160 / 160 / 16$ vs D-QUARK vs PHOTON-160/36/36, SPONGENT-224/224/16 vs S-QUARK vs PHOTON$224 / 32 / 32$, and SPONGENT-256/256/16 vs PHOTON$256 / 32 / 32)$, SPONGENT provides a considerably smaller circuit size. On the other hand, mainly due to either a small number of rounds or a higher rate (message block size) both QUARK and PHOTON achieve higher throughput.

Next, we present the obtained hardware figures for all of the SPONGENT variants. For the purpose of extensive hardware evaluation we use Synopsys Design Compiler version D-2010.03-SP4 4 and target the HighSpeed UMC $130 \mathrm{~nm}$ CMOS generic process provided by Faraday Technology Corporation (fsc0h_d_tc). The results of all the lightweight implementations are summarized in Fig. 7 It is interesting to observe the wide spectrum of SPONGENT hardware performance which ranges from the smallest implementation, consuming only $738 \mathrm{GE}$, until the fastest versions, which achieve more than $66 \mathrm{kbps}$ at $100 \mathrm{kHz}$.

In order to provide very compact implementations, we first focus on serialized designs. We explore four different datapath sizes $(d)$ for each of the SPONGENT variants and we focus on $d \in\left\{4,8, \frac{b}{2}, b\right\}$. These four implementations are clearly marked in Fig. 7 by the four marker points for each different SPONGENT version. An architecture representing our serialized datapath is depicted in Fig. 6(a). The control logic consists of a single counter for the cycle count and some extra combinational logic to drive the selection signals of the multiplexers. In order to further reduce the area we use so-called scan flip-flops, which act as a combination of two input multiplexer and an ordinary D flip-flop ${ }^{5}$. Instead of providing a reset signal to each flip-flop separately, we use two zero inputs at the multiplexers $M_{1}$ and $M_{2}$ to correctly initialize all the flip-flops. This additionally reduces hardware resources, as the scan flip-flops with a reset input approximately require an additional GE per bit of storage. With $g_{i}$ we denote the value of lCounter $_{b}(i)$ in round $i$. Counter $_{b}(i)$ is implemented as an LFSR as discussed in Section 2.3. The input of the message block $m$, denoted with a dashed line, is omitted in some cases, i.e. $d \geq r$. The pLayer module requires no additional logic except some extra wiring.

Using the most serialized implementation, the smallest

3. Our RTL code and the synthesis scripts are available upon request. 4. KU Leuven owns the Synopsys license.

5. Scan flip-flops are typically used to provide scan-chain based testability of the circuit. Due to the security issues of scan-chain based testing [26], other methods such as Built-In-Self-Test (BIST) are recommended for testing the cryptographic hardware. variant of the SPONGENT family, SPONGENT- $88 / 80 / 8$, can be implemented using only 738 GE. Even the largest member of the family, SPONGENT-256/512/256, consumes only $5.1 \mathrm{kGE}$, while providing 256 bits of preimage and second preimage security, and 128 bits of collision resistance. Though some of this advantage is at the expense of a performance reduction, also less serialized (and, thus, faster) implementations result in area requirements significantly lower than $10 \mathrm{kGE}$. To demonstrate this, we implement all the SPONGENT variants as depicted in Fig. 6(b). Every round now requires a single clock cycle, therefore resulting in faster, yet rather compact designs.

Another courtesy of our proposal is the result of 5 times unrolled design of SPONGENT variants which, all running at the maximum frequency of about $600 \mathrm{MHz}$, provide a throughput between $360 \mathrm{Mbps}$ and $2 \mathrm{Gbps}$ (depending on the variant) and consume between $5 \mathrm{kGE}$ and $48 \mathrm{kGE}$.

While it has been well known that the library choice is crucial for the circuit's performance, its impact on a fair comparison between different designs has not been properly addressed in the literature. In order to highlight its importance, we provide results of SPONGENT implemented using four different standard cell libraries (Table 9). Comparing the worst reported performance (i.e. NANGATE45) with the best performance achieved by the advanced NXP90 standard cell library, we discover up to $70 \%$ difference in area which represents a significant margin (the size is compared using gate equivalences). The main cause of the present variance is a difference in relative cell sizes, which is directly related to the library type. Note that the serial implementation of our designs consists of more than $90 \%$ of sequential logic. A single scan flip-flop consumes at least 7.67 GE in NANGATE45. The same cell consumes $6.25 \mathrm{GE}$ and 6.67 GE in UMC130 and UMC180, respectively. The NXP90 library has significantly smaller flip-flops which are the main area consumers in the case of SPONGENT family, and thus provides a considerably better hardware performance.

\section{Conclusion}

In this work, we have explored the design space of lightweight cryptographic hashing by proposing the family of new hash functions SPONGENT tailored for resource-constrained applications. We consider 5 hash sizes for SPONGENT - ranging from the ones offering mainly preimage resistance only to those complying to (a subset of) SHA-2 and SHA-3 parameters. For each parameter set, we instantiate SPONGENT using up to three competing security paradigms (all of them offering full collision security): reduced second-preimage security, reduced preimage and second-preimage security, as well as full preimage and second-preimage security. Each parametrization accounts for its unique implementation properties in terms of ASIC hardware footprint, performance and time-area product, which are analyzed in the 
TABLE 8

Hardware performance of the SPONGENT family and comparison with state-of-the-art lightweight hash designs using NANGATE45, an open core library (post-synthesis results). The nominal frequency of $100 \mathrm{kHz}$ is assumed in all cases and the power consumption is therefore estimated accordingly.

\begin{tabular}{|c|c|c|c|c|c|c|c|c|c|c|}
\hline Hash function & \multicolumn{3}{|c|}{ Security (bit) } & $\begin{array}{c}\text { Hash } \\
\text { (bit) }\end{array}$ & Cycles & $\begin{array}{l}\text { Datapath } \\
\text { (bit) }\end{array}$ & $\begin{array}{c}\text { Process } \\
(\mathrm{nm})\end{array}$ & $\begin{array}{l}\text { Area } \\
\text { (GE) }\end{array}$ & $\begin{array}{l}\text { Throughput } \\
\text { (kbps) }\end{array}$ & $\begin{array}{c}\text { Power* }^{*} \\
(\mu \mathrm{W})\end{array}$ \\
\hline SPONGENT-88/80/8 & 80 & 40 & 40 & 88 & $\begin{array}{c}990 \\
45\end{array}$ & $\begin{array}{c}4 \\
88\end{array}$ & $\begin{array}{l}45 \\
45\end{array}$ & $\begin{array}{c}869 \\
1237\end{array}$ & $\begin{array}{c}0.81 \\
17.78\end{array}$ & $\begin{array}{l}16.50 \\
38.74\end{array}$ \\
\hline SPONGENT-88/176/88 & 88 & 44 & 88 & 88 & $\begin{array}{c}8910 \\
135\end{array}$ & $\begin{array}{c}4 \\
264\end{array}$ & $\begin{array}{l}45 \\
45\end{array}$ & $\begin{array}{l}2264 \\
3633\end{array}$ & $\begin{array}{c}0.99 \\
65.19\end{array}$ & $\begin{array}{c}33.33 \\
140.54\end{array}$ \\
\hline SPONGENT-128/128/8 & 120 & 64 & 64 & 128 & $\begin{array}{c}2380 \\
70\end{array}$ & $\begin{array}{c}4 \\
136\end{array}$ & $\begin{array}{l}45 \\
45\end{array}$ & $\begin{array}{l}1257 \\
1831\end{array}$ & & $\begin{array}{l}21.12 \\
53.21\end{array}$ \\
\hline SPONGENT-128/256/128 & 128 & 64 & 128 & 128 & $\begin{array}{c}18720 \\
195\end{array}$ & $\begin{array}{c}4 \\
384\end{array}$ & $\begin{array}{l}45 \\
45\end{array}$ & $\begin{array}{l}3183 \\
5715\end{array}$ & $\begin{array}{c}0.68 \\
65.64\end{array}$ & $\begin{array}{c}44.64 \\
232.70\end{array}$ \\
\hline SPONGENT-160/160/16 & 144 & 80 & 80 & 160 & $\begin{array}{c}3960 \\
90\end{array}$ & $\begin{array}{c}4 \\
176\end{array}$ & $\begin{array}{l}45 \\
45\end{array}$ & $\begin{array}{l}1572 \\
2406\end{array}$ & & $\begin{array}{l}24.55 \\
73.46\end{array}$ \\
\hline SPONGENT-160/160/80 & 80 & 80 & 80 & 160 & $\begin{array}{c}7200 \\
120\end{array}$ & $\begin{array}{c}4 \\
240\end{array}$ & $\begin{array}{l}45 \\
45\end{array}$ & $\begin{array}{l}2066 \\
3612\end{array}$ & $\begin{array}{l}1.11 \\
66.67\end{array}$ & $\begin{array}{c}30.33 \\
143.41\end{array}$ \\
\hline SPONGENT-160/320/160 & 160 & 80 & 160 & 160 & $\begin{array}{c}28800 \\
240\end{array}$ & $\begin{array}{c}4 \\
480\end{array}$ & $\begin{array}{l}45 \\
45\end{array}$ & $\begin{array}{l}3931 \\
7163\end{array}$ & $\begin{array}{c}0.56 \\
66.67\end{array}$ & $\begin{array}{c}54.36 \\
282.95\end{array}$ \\
\hline SPONGENT-224/224/16 & 208 & 112 & 112 & 224 & $\begin{array}{c}7200 \\
120\end{array}$ & $\begin{array}{c}4 \\
240\end{array}$ & $\begin{array}{l}45 \\
45\end{array}$ & $\begin{array}{l}2070 \\
3220\end{array}$ & & $\begin{array}{l}31.35 \\
96.02\end{array}$ \\
\hline SPONGENT-224/224/112 & 112 & 112 & 112 & 224 & $\begin{array}{c}14280 \\
170\end{array}$ & $\begin{array}{c}4 \\
336\end{array}$ & $\begin{array}{l}45 \\
45\end{array}$ & $\begin{array}{l}2827 \\
4611\end{array}$ & $\begin{array}{c}0.78 \\
65.88\end{array}$ & $\begin{array}{c}41.18 \\
182.96\end{array}$ \\
\hline SPONGENT-224/448/224 & 224 & 112 & 224 & 224 & $\begin{array}{c}57120 \\
340\end{array}$ & $\begin{array}{c}4 \\
672\end{array}$ & $\begin{array}{l}45 \\
45\end{array}$ & $\begin{array}{l}5430 \\
9751\end{array}$ & $\begin{array}{l}0.39 \\
65.88\end{array}$ & $\begin{array}{c}72.32 \\
429.50\end{array}$ \\
\hline SPONGENT-256/256/16 & 240 & 128 & 128 & 256 & $\begin{array}{c}9520 \\
140\end{array}$ & $\begin{array}{c}4 \\
272\end{array}$ & $\begin{array}{l}45 \\
45\end{array}$ & $\begin{array}{l}2323 \\
3639\end{array}$ & $\begin{array}{c}0.17 \\
11.43\end{array}$ & $\begin{array}{c}34.21 \\
109.91\end{array}$ \\
\hline SPONGENT-256/256/128 & 128 & 128 & 128 & 256 & $\begin{array}{c}18720 \\
195\end{array}$ & $\begin{array}{c}4 \\
384\end{array}$ & $\begin{array}{l}45 \\
45\end{array}$ & $\begin{array}{l}3183 \\
5713\end{array}$ & $\begin{array}{c}0.68 \\
65.64\end{array}$ & $\begin{array}{c}44.65 \\
232.32\end{array}$ \\
\hline SPONGENT-256/512/256 & 256 & 128 & 256 & 256 & $\begin{array}{c}73920 \\
385\end{array}$ & $\begin{array}{c}4 \\
768\end{array}$ & $\begin{array}{l}45 \\
45\end{array}$ & $\begin{array}{c}6163 \\
10778\end{array}$ & $\begin{array}{c}0.35 \\
66.49\end{array}$ & $\begin{array}{c}81.85 \\
447.78\end{array}$ \\
\hline РнотоN-80/20/16 [14] & 64 & 40 & 40 & 80 & $\begin{array}{l}708 \\
132\end{array}$ & $\begin{array}{c}4 \\
20\end{array}$ & $\begin{array}{l}45 \\
45\end{array}$ & $\begin{array}{l}1067 \\
1567\end{array}$ & & $\begin{array}{l}14.00 \\
39.92\end{array}$ \\
\hline РнотоN-128/16/16 [14] & 112 & 64 & 64 & 128 & $\begin{array}{l}996 \\
156\end{array}$ & $\begin{array}{c}4 \\
24\end{array}$ & $\begin{array}{l}45 \\
45\end{array}$ & $\begin{array}{l}1394 \\
2172\end{array}$ & $\begin{array}{c}1.61 \\
10.26\end{array}$ & $\begin{array}{l}17.20 \\
49.62\end{array}$ \\
\hline РнOTON-160/36/36 [14] & 124 & 80 & 80 & 160 & $\begin{array}{c}1332 \\
180\end{array}$ & $\begin{array}{c}4 \\
28\end{array}$ & $\begin{array}{l}45 \\
45\end{array}$ & $\begin{array}{l}1741 \\
2849\end{array}$ & $\begin{array}{c}2.70 \\
20.00\end{array}$ & $\begin{array}{l}19.37 \\
65.78\end{array}$ \\
\hline РнотоN-224/32/32 [14 & 192 & 112 & 112 & 224 & $\begin{array}{c}1716 \\
204\end{array}$ & $\begin{array}{c}4 \\
32\end{array}$ & $\begin{array}{l}45 \\
45\end{array}$ & $\begin{array}{l}2142 \\
3586\end{array}$ & $\begin{array}{c}1.86 \\
15.69\end{array}$ & $\begin{array}{l}22.63 \\
78.82\end{array}$ \\
\hline РнотоN-256/32/32 [14] & 224 & 128 & 128 & 256 & $\begin{array}{l}996 \\
156\end{array}$ & $\begin{array}{c}8 \\
48\end{array}$ & $\begin{array}{l}45 \\
45\end{array}$ & $\begin{array}{l}2675 \\
5335\end{array}$ & $\begin{array}{c}3.21 \\
20.51\end{array}$ & $\begin{array}{c}51.62 \\
248.60\end{array}$ \\
\hline U-QUARK [1] & 120 & 64 & 64 & 128 & $\begin{array}{c}544 \\
68\end{array}$ & $\begin{array}{l}1 \\
8\end{array}$ & $\begin{array}{l}45 \\
45\end{array}$ & $\begin{array}{l}1744 \\
3215\end{array}$ & $\begin{array}{c}1.47 \\
11.76\end{array}$ & $\begin{array}{l}51.24 \\
89.39\end{array}$ \\
\hline D-QUARK [1] & 144 & 80 & 80 & 160 & $\begin{array}{c}704 \\
88\end{array}$ & $\begin{array}{l}1 \\
8\end{array}$ & $\begin{array}{l}45 \\
45\end{array}$ & $\begin{array}{l}2200 \\
3695\end{array}$ & $\begin{array}{c}2.27 \\
18.18\end{array}$ & $\begin{array}{l}58.59 \\
87.74\end{array}$ \\
\hline s-QUARK [1] & 192 & 112 & 112 & 224 & $\begin{array}{c}1024 \\
64\end{array}$ & $\begin{array}{c}1 \\
16\end{array}$ & $\begin{array}{l}45 \\
45\end{array}$ & $\begin{array}{l}3001 \\
6155\end{array}$ & $\begin{array}{c}3.13 \\
50.00\end{array}$ & $\begin{array}{c}81.55 \\
146.01\end{array}$ \\
\hline
\end{tabular}

* Power figures are based on the post-synthesis results and serve for a qualitative comparison only.

article. We also perform security analysis in terms of differential properties, linear distinguishers, and rebound attacks.

\section{ACKNOWLEDGMENTS}

Andrey Bogdanov is a postdoctoral fellow of the Fund for Scientific Research - Flanders (FWO). This work is supported in part by the Flemish Government, FWO G.0550.12N and by the Hercules Foundation AKUL/11/19, by the European Commission under contract numbers ICT-2007-216676 ECRYPT NoE phase II, by KU Leuven-BOF (OT/08/027) and by the Research Council of KU Leuven: GOA 11/007 TENSE.

\section{REFERENCES}

[1] Aumasson, J.P., Henzen, L., Meier, W., Naya-Plasencia, M.: Quark: A Lightweight Hash. In: Mangard, S., Standaert, F.X. (eds.) CHES. LNCS, vol. 6225, pp. 1-15. Springer (2010)

[2] Bertoni, G., Daemen, J., Peeters, M., Van Assche, G.: On the Indifferentiability of the Sponge Construction. In: Smart, N.P. (ed.) EUROCRYPT'08. LNCS, vol. 4965, pp. 181-197. Springer (2008)

[3] Bertoni, G., Daemen, J., Peeters, M., Van Assche, G.: Sponge-Based Pseudo-Random Number Generators. In: Mangard, S., Standaert, F.X. (eds.) CHES. LNCS, vol. 6225, pp. 33-47. Springer (2010)

[4] Bogdanov, A., Knezevic, M., Leander, G., Toz, D., Varici, K., Verbauwhede, I.: SPONGENT: A Lightweight Hash Function. In: Preneel, B., Takagi, T. (eds.) CHES'11. LNCS, vol. 6917, pp. 312325. Springer (2011)

[5] Bogdanov, A., Knudsen, L.R., Leander, G., Paar, C., Poschmann, A., Robshaw, M.J.B., Seurin, Y., Vikkelsoe, C.: PRESENT: An Ultra- 


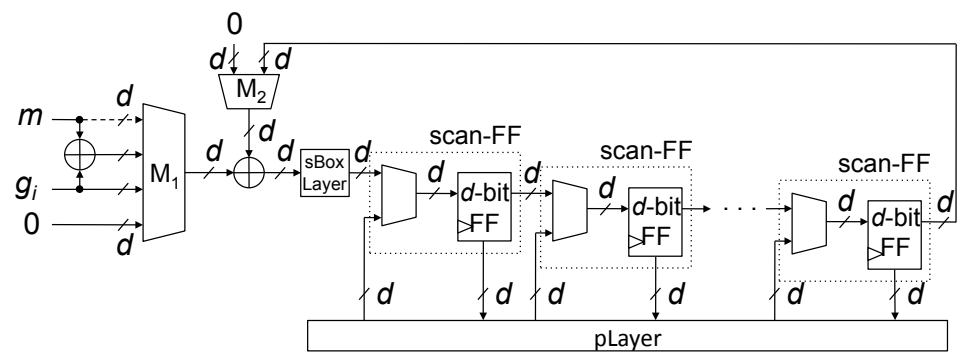

(a)

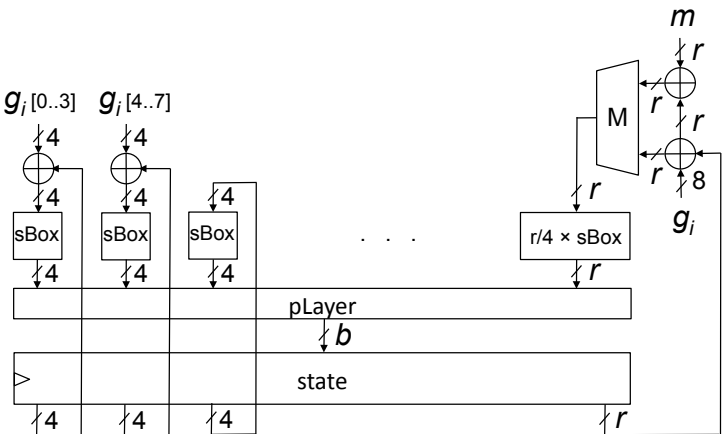

(b)

Fig. 6. Hardware architecture representing (a) serial datapath (b) parallel datapath of the SPONGENT variants.

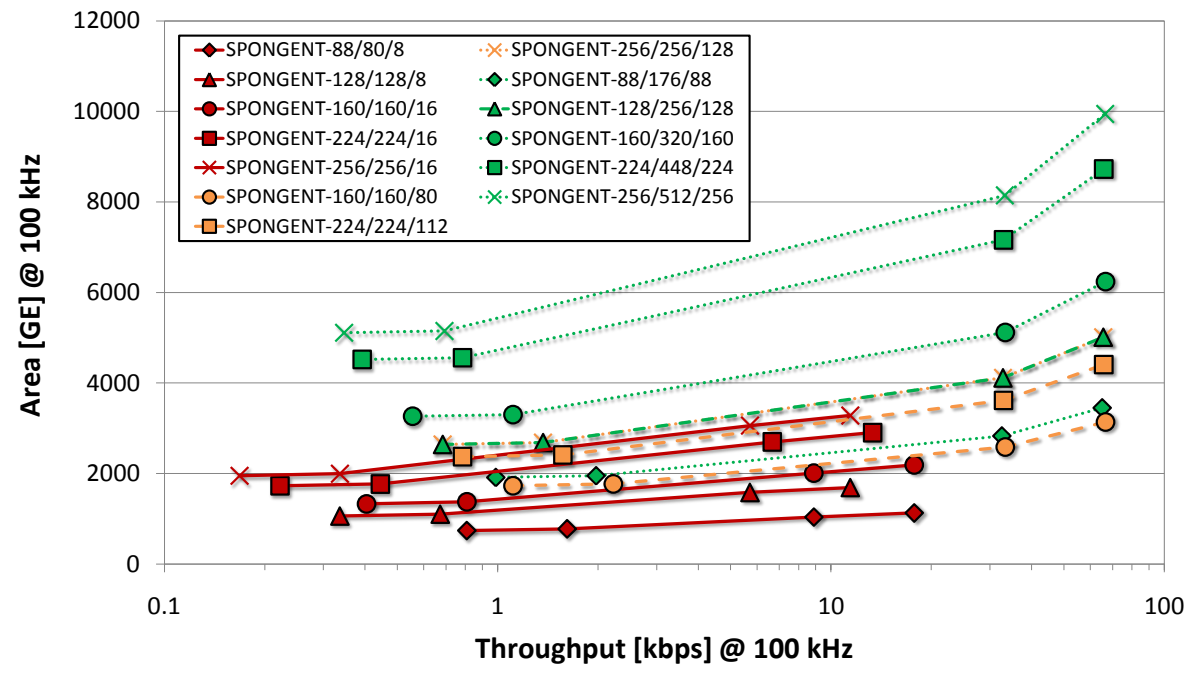

Fig. 7. Area versus throughput trade-off of the SPONGENT hash family (UMC $130 \mathrm{~nm}$ CMOS, post-synthesis results).

Lightweight Block Cipher. In: Paillier, P., Verbauwhede, I. (eds.) CHES'07. LNCS, vol. 4727, pp. 450-466. Springer (2007)

[6] Bogdanov, A., Leander, G., Paar, C., Poschmann, A., Robshaw, M.J.B., Seurin, Y.: Hash Functions and RFID Tags: Mind the Gap. In: Oswald, E., Rohatgi, P. (eds.) CHES'08. LNCS, vol. 5154, pp. 283-299. Springer (2008)

[7] Cho, J.Y.: Linear Cryptanalysis of Reduced-Round PRESENT In: Pieprzyk, J. (ed.) CT-RSA'10. LNCS, vol. 5985, pp. 302-317. Springer (2010)

[8] Collard, B., Standaert, F.X.: A Statistical Saturation Attack against the Block Cipher PRESENT. In: Fischlin, M. (ed.) CT-RSA'09. LNCS, vol. 5473, pp. 195-210. Springer (2009)

[9] Daemen, J., Peeters, M., Van Assche, G.: Sponge Functions. Ecrypt Hash Workshop 2007 (2007), http://www.csrc.nist.gov/ pki/HashWorkshop/PublicComments/2007May.html

[10] De Cannière, C.: Trivium: A Stream Cipher Construction Inspired by Block Cipher Design Principles. In: Katsikas, S.K., Lopez, J., Backes, M., Gritzalis, S., Preneel, B. (eds.) ISC'06. LNCS, vol. 4176, pp. 171-186. Springer (2006)

[11] De Cannière, C., Dunkelman, O., Knežević, M.: KATAN and KTANTAN - A Family of Small and Efficient Hardware-Oriented Block Ciphers. In: Clavier, C., Gaj, K. (eds.) CHES'09. LNCS, vol. 5747, pp. 272-288. Springer (2009)

[12] Duc, A., Guo, J., Peyrin, T., Wei, L.: Unaligned Rebound Attack - Application to Keccak. Cryptology ePrint Archive, Report 2011/420 (2011), http:/ /eprint.iacr.org/2011/420

[13] Gauravaram, P., Knudsen, L.R., Matusiewicz, K., Mendel, F.,
Rechberger, C., Schläffer, M., Thomsen, S.S.: Grøstl - a SHA-3 candidate. Submission to NIST (Round 3) (2011), http://www. groestl.info/Groestl.pdf

[14] Guo, J., Peyrin, T., Poschmann, A.: The PHOTON Family of Lightweight Hash Functions. In: Rogaway, P. (ed.) CRYPTO. LNCS, vol. 6841, pp. 222-239. Springer (2011)

[15] Guo, X., Schaumont, P.: The Technology Dependence of Lightweight Hash Implementation Cost. ECRYPT Workshop on Lightweight Cryptography (2011)

[16] Hein, D.M., Wolkerstorfer, J., Felber, N.: ECC Is Ready for RFID - A Proof in Silicon. In: Avanzi, R.M., Keliher, L., Sica, F. (eds.) SAC'08. LNCS, vol. 5381, pp. 401-413. Springer (2008)

[17] Henzen, L., Aumasson, J.P., Meier, W., Phan, R.C.W.: VLSI Characterization of the Cryptographic Hash Function BLAKE. http: //131002.net/data/papers/HAMP10.pdf (2010)

[18] Kim, M., Ryou, J., Jun, S.: Etficient Hardware Architecture of SHA256 Algorithm for Trusted Mobile Computing. In: Yung, M., Liu, P., Lin, D. (eds.) Inscrypt. LNCS, vol. 5487, pp. 240-252. Springer (2008)

[19] Leander, G.: On linear hulls, statistical saturation attacks, present and a cryptanalysis of puffin. In: Paterson, K.G. (ed.) EUROCRYPT'11. LNCS, vol. 6632, pp. 303-322. Springer (2011)

[20] Leander, G., Abdelraheem, M.A., AlKhzaimi, H., Zenner, E.: A Cryptanalysis of PRINTcipher: The Invariant Subspace Attack. In: Rogaway, P. (ed.) CRYPTO. LNCS, vol. 6841, pp. 206-221. Springer (2011)

[21] Mendel, F., Rechberger, C., Schläffer, M., Thomsen, S.S.: The 
TABLE 9

Area of the SPONGENT family compared using four different standard cell libraries (post-synthesis results).

The nominal frequency of $100 \mathrm{kHz}$ is assumed in all cases.

\begin{tabular}{l|c|cccc}
\hline \multirow{2}{*}{ Hash function } & $\begin{array}{c}\text { Datapath } \\
\text { (bit) }\end{array}$ & UMC & UMC & NANGATE & NXP \\
& & $130 \mathrm{~nm}$ & $180 \mathrm{~nm}$ & $45 \mathrm{~nm}$ & $90 \mathrm{~nm}$ \\
\hline \multirow{2}{*}{ SPONGENT-88/80/8 } & 4 & 738 & 759 & 869 & 521 \\
& 88 & 1127 & 1232 & 1237 & 883 \\
SPONGENT-88/176/88 & 4 & 1912 & 1965 & 2264 & 1308 \\
& 264 & 3450 & 3847 & 3633 & 2553 \\
\hline \multirow{2}{*}{ SPONGENT-128/128/8 } & 4 & 1060 & 1103 & 1257 & 737 \\
& 136 & 1687 & 1855 & 1831 & 1279 \\
SPONGENT-128/256/128 & 4 & 2641 & 2724 & 3183 & 1813 \\
& 384 & 5011 & 5581 & 5715 & 4167 \\
\hline \multirow{2}{*}{ SPONGENT-160/160/16 } & 4 & 1329 & 1367 & 1572 & 918 \\
& 176 & 2190 & 2241 & 2406 & 1752 \\
SPONGENT-160/160/80 & 4 & 1730 & 1769 & 2066 & 1192 \\
& 240 & 3139 & 3434 & 3612 & 2650 \\
SPONGENT-160/320/160 & 4 & 3264 & 3340 & 3931 & 2232 \\
& 480 & 6237 & 6949 & 7163 & 5262 \\
\hline \multirow{2}{*}{ SPONGENT-224/224/16 } & 4 & 1728 & 1768 & 2070 & 1192 \\
& 240 & 2903 & 3203 & 3220 & 2334 \\
SPONGENT-224/224/112 & 4 & 2371 & 2422 & 2827 & 1621 \\
SPONGENT-224/448/224 & 336 & 4406 & 4900 & 4611 & 3197 \\
& 4 & 4519 & 4625 & 5430 & 3069 \\
SPONGENT-256/256/16 & 4 & 8726 & 9696 & 9751 & 6932 \\
\hline \multirow{2}{*}{ SPONGENT-256/256/128 } & 272 & 3281 & 3721 & 3639 & 2612 \\
& 384 & 2641 & 2724 & 3183 & 1813 \\
& 4 & 5110 & 5232 & 5713 & 4213 \\
& 768 & 9944 & 11054 & 10778 & 7426 \\
\hline
\end{tabular}

Rebound Attack: Cryptanalysis of Reduced Whirlpool and Grøstl. In: Dunkelman, O. (ed.) FSE'09. LNCS, vol. 5665, pp. 260-276. Springer (2009)

[22] NANGATE: The NanGate 45nm Open Cell Library, available at http://www.nangate.com

[23] Rohde, S., Eisenbarth, T., Dahmen, E., Buchmann, J., Paar, C.: Fast Hash-Based Signatures on Constrained Devices. In: Grimaud, G., Standaert, F.X. (eds.) CARDIS'08. LNCS, vol. 5189, pp. 104-117. Springer (2008)

[24] Tillich, S., Feldhofer, M., Issovits, W., Kern, T., Kureck, H., Muehlberghuber, M., Neubauer, G., Reiter, A., Koefler, A., Mayrhofer, M.: Compact Hardware Implementations of the SHA3 Candidates ARIRANG, BLAKE, Grøstl, and Skein. Cryptology ePrint Archive, Report 2009/349 (2009)

[25] Van Assche, G.: Errata for Keccak presentation. E-mail sent to the NIST SHA-3 mailing list on Feb 7 2011, on behalf of the Keccak team (2011)

[26] Yang, B., Wu, K., Karri, R.: Scan Based Side Channel Attack on Dedicated Hardware Implementations of Data Encryption Standard. International Test Conference pp. 339-344 (2004)

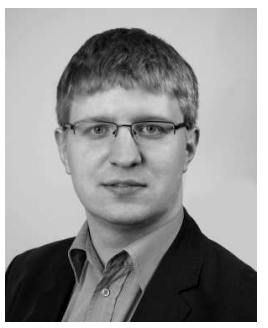

Andrey Bogdanov is currently an FWOsupported post-doctoral researcher at the Department of Electrical Engineering of KU Leuven in Belgium. He holds a Ph.D. in Electrical Engineering and Computer Science from Ruhr University Bochum, Germany (2009). In 2011, he was Visiting Researcher with Microsoft Research. His interests include cryptanalysis and design of symmetric-key algorithms as well as aspects of provable security and side-channel analysis.

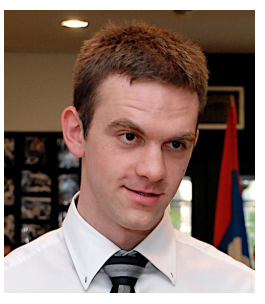

Miroslav Knežević is currently a security researcher at NXP Semiconductors. He received his M.S. degree from the University of Belgrade, Serbia, in 2006, and his Ph.D. degree in electrical engineering from the KU Leuven, Belgium, in 2011. His main research interest includes computer arithmetic and efficient implementations of symmetric-key and public-key cryptographic algorithms.

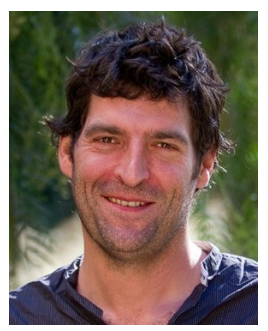

Gregor Leander is currently an associate professor at the Technical University Denmark. $\mathrm{He}$ received his $\mathrm{PhD}$ in mathematics from Ruhr University Bochum, Germany in 2004. His research interests include design and analysis of symmetric cryptography and Boolean functions.

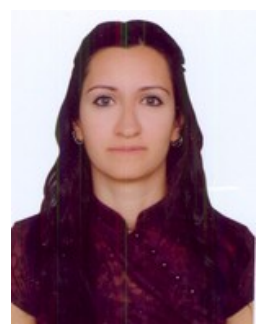

Deniz Toz is currently a Ph.D. student at the Department of Electrical Engineering of KU Leuven in Belgium. She has masters degree in Cryptography from Middle East Technical University, Ankara, Turkey. Her research interests are symmetric-key components. Mainly, analysis and design of symmetric-key primitives, and hash functions.

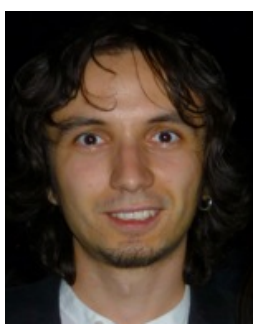

Kerem Varıcı is currently a Ph.D. student at the Department of Electrical Engineering of KU Leuven in Belgium. He had masters degree in Cryptography from Middle East Technical University, Turkey in 2008. His research interests include analysis and the design of the symmetric-key algorithms.

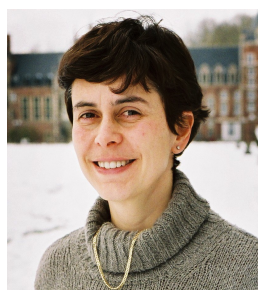

Ingrid Verbauwhede Ingrid Verbauwhede is a Professor at the KU Leuven, Belgium. She joined the research group COSIC in 2003, and she leads the embedded systems and hardware group of it. Before that, from 1998 she was an associate professor at UCLA, Los Angeles, $\mathrm{CA}$, where she founded the EMSEC (embedded security) research group. In 2005 she became a adjunct professor at UCLA. Ingrid received her electrical engineering degree and her Ph.D. degree from KULeuven. She was a post-doctoral visiting researcher and lecturer at UC Berkeley and worked for TCSI and Atmel in Berkeley, CA. She is a Member of IACR and a Senior Member of IEEE. She was elected member of the Royal Flemish Academy of Belgium for Science and the Arts in 2011. Her main interest is in the design and the design methods for secure embedded circuits and systems. 